Volumen: 23 Fecha de recibido: 11/8/2020 Fecha de publicación: Enero-Junio de 2021

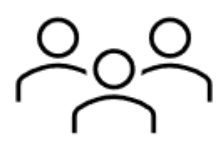

Año: 2021 Fecha de aceptado: 25/9/2020 Correo: revista.societas@up.ac.pa

Numero: $1 \quad$ Número de Páginas: 1-29

URL:https://revistas.up.ac.pa/index.php/societas

\title{
Tecnologías 4.0: El Desafío De La Educación Media En Colombia
}

\author{
Alejandra Fonseca Camargo' ${ }^{1}$. alejandrafo@hotmail.com \\ Luz Stella Ahumada Méndez². luzstellaahumada@gmail.com \\ Universidad Metropolitana de Educación Ciencia y Tecnología. Facultad de \\ Educación, Panamá, Panamá
}

\section{Resumen}

La cuarta revolución industrial (4RI) llegó para transformar la manera como se desarrollan las áreas productivas, económicas y comerciales; esto implica un cambio en la manera de vivir, trabajar y de relacionarse con los otros; lo anterior ha generado a nivel de las organizaciones una preocupación por la forma en que se asumirán retos como la globalización y los cambios tecnológicos; poniendo al descubierto la necesidad de realizar estudios relacionados con el incremento de la productividad, la competitividad y los procesos de comercialización, mediante la implementación de tecnologías 4.0; este artículo es una reflexión acerca del desafío que representa para el ciclo de educación media pública en Colombia, introducir las tecnologías de 4RI en las aulas, no solo como medio para facilitar el aprendizaje, sino como objeto de estudio; de modo que, los estudiantes desarrollen habilidades que les permitan dejar de ser consumidores de productos tecnológicos, para convertirse en creadores de nuevas tecnologías ampliando su

\footnotetext{
${ }^{1}$ Estudiante de Doctorado en Ciencias de la Educación con énfasis en Investigación, Evaluación y Formulación de Proyectos Educativos en la Universidad Metropolitana de Educación, Ciencia y Tecnología - UMECIT. Magister en Educación de la Universidad Santo Tomás Bogotá - Colombia. Ingeniero de Sistemas de la Universidad de Cundinamarca sede Fusagasugá - Colombia. Email:alejandrafo@hotmail.com.

${ }^{2}$ Doctora en Educación Social de la Universidad de Granada España, Magíster en Educación, de la Universidad Pedagógica Nacional Bogotá - Colombia. Docente Doctorado del programa de Educación de la Universidad Metropolitana de Educación, Ciencia y Tecnología - UMECIT . E-mail: luzstellaahumada@gmail.com
} 
horizonte académico y laboral. La metodología empleada para la elaboración del presente escrito fue la interpretación hermenéutica de fuentes bibliográficas y de investigación respecto a la $4 \mathrm{Rl}$ y su incidencia en el ámbito educativo; los hallazgos muestran una clara necesidad de transformar el currículo actual del área de Tecnología e Informática en el ciclo de educación media en Colombia.

Palabras clave: Cuarta revolución industrial, tecnologías 4.0, habilidades 4RI, estándares de competencias, Tecnología e Informática, Educación Media.

\begin{abstract}
The Fourth Industrial Revolution phenomenon (4IR) has come to change how manufacturing, economy and commerce work; it means a new way to live, to keep a job and to stablish relationships with others. All this has produced in an organizational level, a genuine preoccupation about how to confront challenges like globalization and technological changes; consequently it has revealed the need to investigate how to improve productivity, competitiveness and distribution chains, using 4.0 technologies; this paper has considered the big challenge of including technologies 4IR inside of medium public education classrooms in Colombia, not just using them as teaching resources but consider them more like study objects; in order to improve technological students skills that allows them to stop being technology consumers, to become creators of new technologies and get better academic and job opportunities. The methodology used in this paper was hermeneutical analysis of bibliographical sources and related research results about 4IR and its impact in the education field; the results show a clear requirement of changing the actual curriculum of Technology and computing class in the medium education level in Colombia.
\end{abstract}

Keywords: Fourth industrial revolution, technology 4.0, 4IR skills, Standard Skills, Technology and Computing, medium education level. 


\section{Introducción}

En momentos de crisis como el actual, mucho se ha hablado de las tecnologías 4.0 como elemento fundamental para darle continuidad o en su defecto reinventar las actividades económicas, mantener contacto humano sin ponerse en riesgo y desarrollar procesos educativos sin exponer a los estudiantes; la expresión tecnologías 4.0 , se emplea para referirse a tecnologías de cuarta generación, también denominadas tecnologías de cuarta revolución industrial, que agrupan una serie de elementos de hardware y de software con el potencial de modificar las formas de fabricación y producción tradicionales y que permiten la digitalización de procesos, transformando radicalmente la manera en que se hacen las cosas.

El término cuarta revolución industrial (4RI) fue empleado por primera vez por Klaus Schwab en el Foro Económico Mundial celebrado en Davos-Suiza en el año 2016, para referirse a las tecnologías que permiten digitalizar y conectar diferentes actividades humanas (Miller, 2016); sin embargo, éste tiene su origen en una estrategia del gobierno alemán llamada Industria 4.0 propuesta en el año 2011 en la feria de Hannover; el objetivo consistía en lograr una transformación de la producción industrial mediante la digitalización y la apropiación de las nuevas tecnologías como loT (internet de las cosas), integración de procesos técnicos con la comercialización, el mapeo digital, la virtualización del mundo real y la creación de fábricas inteligentes con producción inteligente(Rojko, 2017).

La industria 4.0 significó para las empresas alemanas redes inteligentes de productos y procesos en la creación de valor industrial; se prevé que esta estrategia para el año 2025 aumentará el potencial de crecimiento anual en los diferentes sectores de la economía hasta en un 2.2\% (Bauer, Schlund, Marrenbach y Ganschar, 2014); las perspectivas de éxito y los logros alcanzados hasta el momento, han generado revuelo en los gobiernos y el empresariado a nivel global, de modo que, se ha llegado a la formulación de políticas estatales y estrategias de mercado tendientes a sacar el mayor provecho posible de la cuarta revolución industrial (Liao, Rocha, Deschamps, Brezinski y Venâncio, 2018). 
Más allá de los cambios en las formas de producción y obtención de los bienes y servicios, Schwab (2016) plantea que la cuarta revolución industrial producirá un cambio abrupto y radical tanto en los sistemas económicos como en las estructuras sociales; si bien la primera revolución industrial(1784) impulsó la producción mecánica con la invención de la máquina de vapor; la segunda (1870) hizo posible la producción en masa con el advenimiento de la electricidad y la tercera(1969) desarrolló la era informática con la aparición del computador, lo cual permitió programar máquinas y redes, favoreciendo la automatización; la cuarta revolución que aparece desde inicios del siglo XXI se ha centrado en la revolución digital, se caracteriza por la fusión de tecnologías como la robótica, la nanotecnología, la secuenciación genética y la computación cuántica entre otras ya existentes, para lograr dominios físicos, digitales y biológicos que tendrán un efecto disruptivo e innovador en todos los campos de la actividad humana.

Estudios en torno a las tecnologías predominantes durante la 4RI (Alonso Calpeño, Santander - Castillo, Ramirez - Chocolatl y Alanis - Teutle , 2019; C Glenn, Florescu y Millenium Project Team, 2016; González García, 2018; Mantilla Avendaño y González-Pérez, 2015) han establecido que durante esta etapa, el internet de las cosas (IOT), los sitios web, las aplicaciones móviles, la robótica, la nanotecnología, la Blockchain, la ciberseguridad, la Big data, el transporte autónomo, la inteligencia artificial, la realidad aumentada, la realidad virtual, la impresión 3D, la biotecnología y los electrónicos vestibles, serán las tecnologías que tendrán mayor desarrollo y aplicación en los diferentes aspectos que afectan la vida de las personas.

Las tecnologías antes mencionadas no son nuevas; de hecho, algunas de ellas llevan varias décadas en desarrollo; sin embargo, se prevé que durante la cuarta revolución industrial estas se integrarán para mejorar su funcionalidad y automatizar los procesos (Rose, 2016); su fusión e inter operatividad permitirán digitalizar a una mayor escala la forma tradicional de desarrollar tareas 0 interactuar con los demás.

Según Schwab (2016), el impacto de la cuarta revolución industrial podrá sentirse en la economía, en los negocios, en la forma de gobernar, en la 
organización de ciudades, regiones y países, en la manera como se organizan las sociedades, en la manera como se distribuyen los recursos, en el sentido de identidad que construyen los individuos, en la forma como se conectan entre sí las personas y se maneja la información tanto pública como privada; se plantea la necesidad de prepararse para esta nueva fase de desarrollo trabajando en dos frentes específicos: la adopción de estrategias desde el gobierno y la industria para hacer parte del cambio tecnológico y lograr su mejor aprovechamiento y la adaptación del sistema educativo para ofrecer conocimientos y habilidades contextualizadas con la 4RI.

La postura de Schawb (2016), así como los hallazgos de otros estudios realizados en relación a las habilidades que el sistema educativo debe desarrollar en los estudiantes de cara a la cuarta revolución industrial y la consecuente transformación del empleo, así como la desaparición de algunas ocupaciones debido a la automatización de las mimas (Hecklau, Flachs y Khol, 2016; UNESCO, 2005; WEF, 2018; Eberhard et al., 2017; Lubis, Abash y Lumbanraja, 2019; Manyika et al., 2017; Manpower Group, 2018; Romero et al., 2016; Frey y Osborne, 2013; Dombrowski y Wagner, 2014), permiten vislumbrar que los conocimientos y la acreditación de títulos académicos, no serán en adelante un elemento determinante en la cualificación de un individuo; a cambio, tendrán un mayor valor sus habilidades para comunicarse, cooperar, trabajar en equipo, resolver problemas, tomar decisiones, adaptarse a los cambios, crear e innovar, pensar de una manera sostenible, compartir sus conocimientos y aprender por su propia cuenta.

Lo referido anteriormente, es una consecuencia de la democratización del conocimiento, derivada de la accesibilidad al mismo en los medios electrónicos de información; en la actualidad, quien se interese por determinado tema, puede lograr aprendizajes en relación al mismo, empleando recursos electrónicos que se encuentran disponibles inclusive de forma gratuita en la red; por esto, más allá de enseñar conocimientos acerca de un área en específico, la meta del sistema educativo es formar habilidades que le permitan al individuo aprender de manera 
autónoma y emplear esos aprendizajes asertivamente en diferentes entornos y situaciones.

Las habilidades tecnológicas requeridas en la cuarta revolución industrial, modifican sustancialmente lo que se espera del sistema educativo; no sólo en términos de la capacidad de utilización de tecnologías 4.0, sino también en relación al uso de esos recursos para la solución de problemas reales y el diseño nuevas tecnologías tanto de hardware como de software.

Lo antes expresado, redefine las metas de aprendizaje que actualmente el sistema educativo colombiano plantea para la enseñanza del área de Tecnología e Informática en la educación media y establece la necesidad de evaluar la pertinencia de los estándares educativos planteados por el Ministerio de Educación, así como la forma como éstos se desarrollan actualmente en las aulas.

El presente escrito tiene como finalidad analizar la trayectoria que ha seguido el sistema educativo colombiano en el ciclo de educación media, en relación a la enseñanza en tecnología y el desafío que enfrenta para contextualizar estas prácticas educativas con el fenómeno de la cuarta revolución industrial.

\section{Definición del término tecnologías 4.0}

Hablar de tecnología es hacer referencia a diversos elementos e instrumentos que se utilizan cotidianamente para facilitar tareas o hacer más cómoda la vida de las personas; regularmente, suele pensarse que tecnología está representada en dispositivos complejos cuyo diseño y construcción requirieron de conocimientos y habilidades avanzadas; segregando así de esta categoría aquellos artefactos cuya estructura sencilla y funcionalidad resuelve problemas comunes.

Pese a lo anterior, cuando se habla de tecnología, se están abarcando infinidad de elementos, artefactos y dispositivos que permitieron satisfacer una necesidad humana o resolver un problema cotidiano; Rodríguez (1998) define la tecnología "como el conjunto de saberes inherentes al diseño y concepción de los instrumentos (artefactos, sistemas, procesos y ambientes) creados por el hombre 
a través de su historia para satisfacer sus necesidades y requerimientos personales y colectivos" (p.114).

La tecnología ha existido desde las primeras etapas de la historia de la humanidad y su desarrollo ha hecho posible, no solo la subsistencia humana, sino también la adaptación del medio y los recursos para la conformación de la sociedad y de la cultura, tal como se conocen en la actualidad.

Desde luego, los primeros inventos tecnológicos, aunque trascendentales para sobrevivir, tenían estructuras más sencillas y usos más limitados; así que estas tecnologías serían la versión 1.0 de los desarrollos tecnológicos; en la actualidad se habla de dispositivos mucho más avanzados cuyo diseño y construcción integra diferentes disciplinas; por su nivel de complejidad, se les denomina tecnologías 4.0.

Las tecnologías 4.0 o tecnologías de cuarta revolución industrial son un conjunto de desarrollos tecnológicos que tienen el potencial de transformar las operaciones de mercado y las comunicaciones, mediante la flexibilización de la producción y de las cadenas de suministros para llegar a la manufactura y distribución de productos cuyo diseño se deriva de las opiniones y requerimientos de los consumidores, con un sentido de sostenibilidad y responsabilidad ambiental (Morrar, Arman y Mousa, 2017).

Brian Housseholder (2018) sostiene que esta nueva revolución es un desafío hacia la siguiente fase para cambiar la manera de pensar y de trabajar, con el uso de datos para agregar valor a partir de la implementación de las tecnologías avanzadas del momento, lo esencial, es que los líderes de las diferentes organizaciones estén capacitados para implementar los aportes de la Cuarta Revolución Industrial con el fin de dinamizar las economías, los roles de las personas, trabajos de las diferentes áreas de la industria, para que se puedan tomar decisiones de manera holística y con mayor información, como también se establecen mejores relaciones con los usuarios o clientes en cuanto a los productos y servicios que ellos demandan.

Por consiguiente, estas tecnologías han sido inicialmente concebidas para la industria, pero su campo de aplicación suele transformar todos y cada uno de 
los aspectos de la vida de las personas; es así como los productos que el mercado pone al alcance de los consumidores, terminan generando tendencias y nuevos hábitos de consumo o de comportamiento; estos cambios a su vez, son insumo para la creación o rediseño de nuevos productos o servicios; el rol de las tecnologías 4.0 , es permitir ese dinamismo entre los sectores de producción y consumo casi en tiempo real.

Schwab (2016) y otros autores (Alonso - Calpeño, Santander - Castillo, Ramirez - Chocolatl y Alanis - Teutle , 2019; C Glenn, Florescu y Millenium Project Team, 2016; González García, 2018; Mantilla Avendaño y González -Pérez, 2015), plantean como tecnologías 4.0 o de cuarta revolución industrial las siguientes:

El internet de las cosas, conocido como loT por su sigla en inglés (Internet of Things) es una tecnología en la que se busca conectar máquinas, equipos, software y cosas del entorno a la internet mediante la asignación de una dirección de protocolo de internet única denominada IP, que permite efectuar la conexión sin la intervención humana (Yinghui y Guanyu, 2010); esta tecnología hace posible que los usuarios manipulen dispositivos de manera remota mediante su teléfono móvil u otro terminal con conexión a internet; también permite que un artefacto con IoT, acceda a la internet para reportar novedades, buscar información útil para su funcionamiento o ejecutar determinadas funciones; algunos ejemplos de dispositivos con tecnología loT son las cámaras de vigilancia, los robots de limpieza, los semáforos inteligentes, el alumbrado público o un vehículo autónomo.

Los sitios web, también conocidos como portales web, hacen referencia a un conjunto de páginas web interrelacionadas que se encuentra alojadas en un domino. Una página web es una fuente de información que se ha elaborado como mínimo en lenguaje HTML y que se ha adaptado para la World Wide Web para que pueda ser visualizada a través de un navegador; se compone de diversos archivos como imágenes, videos y sus archivos de código fuente (Equipo Vortice, 2010). Los sitios web son herramientas eficaces para publicar información, interactuar con los usuarios y ofrecer todo tipo de servicios, además, se pueden visualizar en cualquier dispositivo con conexión a internet. 
Las aplicaciones móviles o apps, que son desarrollos de software que funcionan en teléfonos móviles y que se han creado con un fin concreto (Santamaría-Puerto \& Hernández-Rincón, 2015). Las apps son herramientas que permiten ofrecer soluciones prácticas a problemas de todo tipo y se encuentran a disposición de todos los usuarios en tiendas como Play Store, Google Play, App Store o en las plataformas de los creadores de las App; las más comunes a octubre de 2017 eran Facebook, WhatsApp, Chrome, Facebook, Messenger e Instagram (La Nación, 2017).

La Robótica es una disciplina transversal que se interrelaciona con diferentes áreas del conocimiento para definir la arquitectura y funcionamiento de los robots; Reyes, (2011) sostiene que "La Robótica es una disciplina científica que aborda la investigación y desarrollo de una clase particular de sistemas mecánicos, denominados robots manipuladores, diseñados para realizar una amplia variedad de aplicaciones industriales, científicas, domésticas y comerciales" (p.41); como puede verse, los robots tienen aplicación en diferentes campos y se cree que durante la cuarta revolución industrial, serán fundamentales en la automatización de tareas de producción.

La computación en la nube, es también una de las más claras tendencias en la cuarta revolución industrial, debido a los grandes volúmenes de información que se manejan y la necesidad de acceder a ella de forma instantánea; Celaya (2014) define el cloud computing o simplemente cloud, como un conjunto de aplicaciones y servicios informáticos cuya principal característica es que se alojan en internet; el usuario puede acceder a ellos de manera instantánea en cualquier momento y lugar; este servicio no es exclusivo para las empresas, en la actualidad los usuarios usan herramientas de cloud tan comunes como google drive o icloud para almacenar sus archivos personales.

Los grandes volúmenes de datos conocidos como Big data, son sin duda una de las tecnologías más novedosas de la $4 \mathrm{RI}$, ya que ofrecen información que permite hacer predicciones acerca de determinados eventos con base en datos aparentemente inconexos; según Castillo (2018) el Big data son un conjunto de datos de tamaño monstruoso que presentan tres características: gran volumen, 
variedad y se producen a gran velocidad; esto hace que sobrepasen la capacidad del software para capturarlos, gestionarlos y procesarlos, pero que a su vez brindan datos útiles para las empresas; el procesamiento de estos datos llamado data mining (minería de datos) emplea algoritmos de inteligencia artificial que permiten detectar patrones entre los datos para hallar información nueva que revele el comportamiento de determinado fenómeno tanto en el presente como en el futuro.

La inteligencia artificial es definida por su pionero Marvin Minsky (2010) como una ciencia que permite que las máquinas realicen tareas que requieran inteligencia tal como las haría un humano; es una tecnología que busca simular la capacidad de las personas para resolver problemas, tomando decisiones a partir de información relevante y aprendiendo de la experiencia; el fin último de la inteligencia artificial (IA) es emular o superar la inteligencia humana; este propósito que se ha denominado momento de la singularidad, no ha sido posible según Mantaras y Meseguer (2017 debido a la incapacidad de dotar a las máquinas de sentido común, que es el resultado de las experiencias de interacción con el entorno y en el caso de las máquinas, éstas no pueden tener interacciones corpóreas directas con el entorno; por tanto, depende de la robótica u otras tecnologías de 4RI construir hardware que permita construir el sentido común.

La relidad aumentada (RA), es una tecnología cuyo uso es bastante común en la actualidad; Prendes (2015) define la realidad aumentada como "una tecnología que superpone a una imagen real obtenida a través de una pantalla, imágenes, modelos 3D u otro tipo de informaciones generados por ordenador" (pág. 188). La RA se emplea actualmente para imágenes, espacios y lugares; los ejemplos más notables de su uso son los videojuegos, las Apps para sitios turísticos, los filtros para las cámaras de los teléfonos y los mensajes publicitarios.

La realidad virtual o VR por sus siglas en inglés, es una tecnología que intenta emular elementos del mundo real; Para Castañares (2011) la realidad virtual es "lograr una relación interactiva con el sistema de la máquina, de tal manera que el objetivo es la creación de "un entorno mental" en el que se producen unos efectos semejantes a los que produce la realidad"(p.60); esto se 
logra mediante estímulos que generan en el individuo la sensaciones que le permiten integrarse al entorno; Parés y Parés (2010) proponen tres usos posibles para la realidad virtual: los usos sustitutivos donde se busca emular un uso convencional de otro medio, por ejemplo el recorrido virtual de un museo; los usos complementarios que buscan complementar los usos convencionales de otro medio, por ejemplo la presentación virtual de un proyecto de construcción con sus redes internas; por último, los usos originales que intentan sacar provecho de las propiedades de la VR para plantear o resolver problemas por ejemplo un sistema de realidad virtual de ensamblaje intuitivo de moléculas complejas con force feedback.

La ciberseguridad o cybersecurity, son un conjunto de herramientas que garantizan la seguridad de los usuarios a la hora de proteger su información en medios digitales; Navarro, Martínez y Martínez (2018), definen la ciberseguridad como la protección de la infraestructura informática o la información que contiene aplicando protocolos, métodos y reglas para minimizar los riesgos a los que pueda estar sometida; la función de la ciberseguridad es mantener contrarrestar los ciberataques manteniendo la integridad de los datos.

La Blockchain o cadena de bloques, es un protocolo de sistema de pago electrónico entre iguales (peer to peer o P2P), que usa criptomonedas (monedas digitales que no son contraladas por los estados), siendo la más popular de ellas Bitcoin; el protocolo establece normas de computación distribuida que garantiza la integridad de la información; la fiabilidad del sistema se logra mediante el registro de los bloques en secuencia (llamados blockchain o cadena de bloques), en la Blockchain miles de usuarios tienen acceso al registro de cada bloque, lo que permite contrastar su validez y evita centralizar la información para no hacerla susceptible a ciberataques. La blockchain es pública y se protege mediante encriptación (Tapscott y Tapscott, 2017); esta tecnología evita la intervención de intermediarios en las transacciones virtuales y tiene el potencial de reemplazar el dinero físico, así como los medios de pago actuales.

La impresión en 3D, es un sistema tecnológico que permite imprimir un diseño en tres dimensiones; este proceso suele hacerse mediante el uso de 
impresoras 3D; "Una impresora 3D es una máquina que crea objetos de plástico u otros materiales utilizando un proceso de fabricación de aditivos. La fabricación aditiva produce objetos en una sucesión de capas de la parte inferior, hacia arriba"(Himenez y Stratasys, 2011); una impresora 3D se pueden imprimir estructuras con formas geométricas diversas, entrelazadas como tejidos, piezas mecánicas, piezas de joyería, piezas odontológicas, piezas de aviones o autos e incluso órganos humanos; se pueden emplear para la impresión plásticos de diferentes tipos, metales y aleaciones de metales, cerámica y se ha experimentado con otros materiales como alimentos, tejidos humanos o animales e incluso con hormigón (Berchon y Luyt, 2016).

El transporte autónomo, es una tecnología que permite llevar personas u objetos de un lugar a otro, en medios que no requieren conductor humano, pues están dotados de tecnologías que son capaces de imitar las habilidades de manejo y control, percibiendo el medio que le rodea y siguiendo un plan de desplazamiento, mediante la combinación de elementos informáticos tanto de hardware, como de software (Salgado, 2019); los vehículos autónomos emplean sensores externos que permiten construir continuamente un mapa virtual de la ruta que se sigue y aplicar sistemas de frenado en caso de imprevistos; también reciben mediante aplicaciones de GPS información acerca del tránsito y posibles atajos para ahorrar tiempo; esta tecnología ofrece diferentes niveles de apoyo a los conductores, desde simples advertencias hasta una conducción totalmente autónoma.

La biotecnología, que integra el uso de la tecnología con sistemas biológicos con el fin de producir innovaciones en áreas tales como la medicina, la producción de alimentos, la industria y el cuidado del planeta; ProArgentina (2005), define la biotecnología como la combinación de la ciencia y de la ingeniería mediante el uso de orgasnismos vivos para la producción de bienes y servicios que mejoren procesos industriales; en la actualidad la biotecnología ha tenido mucha incidencia en el diseño de tratamientos médicos y vacunas, así como en la creación de materiales biodegradables que reducen la afectación ambiental de la industria. 
Los wearable electronics o tecnología vestible, hace referencia a prendas que se elaboran con textiles inteligentes más conocidos como e-textiles; Stoppa y Chiolerio (2014) los definen como "telas que cuentan con dispositivos electrónicos e interconexiones tejidas en ellos, presentando flexibilidad física y tamaño típico que no se puede lograr con otras técnicas de fabricación electrónica existentes" ( $p$. 11957); Esta tecnología representa la unión de dos técnicas: el diseño de prendas y el diseño de circuitos; la fusión de estas tecnologías, hace posible que las personas integren a su biología (ya que todo el mundo usa ropa) componentes electrónicos en forma de prendas que además de mantener la temperatura corporal, realizan otras funciones que actualmente requieren el uso de algún dispositivo externo, tales como medir el ritmo cardiaco, la temperatura corporal, guardar información o navegar en la internet.

La nanotecnología se refiere a desarrollos tecnológicos con tamaño muy pequeños a nivel de la nanoescala; es decir tamaños cercanos al 0,000000001 metros. Taniguchi (1974) define la nanotecnología como un conjunto de técnicas que permiten fabricar dispositivos tecnológicos con una precisión del orden de 1 nanómetro. La nanotecnología implica que es posible miniaturizar diversos elementos, cuyo tamaño es más conveniente si es microscópico, ejemplo de ello podrían ser dispositivos que permitan tomar muestras médicas, elementos que pudiesen ser introducidos en el cuerpo para tratar enfermedades, dispositivos para descontaminar ríos, desarrollos para descomponer materiales no biodegradables, prótesis para rehabilitación, etc.

Se prevé que las tecnologías antes descritas logren sus puntos máximos de desarrollo durante la cuarta revolución industrial y se implementen en dispositivos de uso diario, de modo que, no estarán presentes exclusivamente en la industria, sino que por el contrario harán parte de la cotidianeidad de las personas, transformando sus costumbres y la forma en que suelen realizar sus actividades tanto laborales como de otras índoles.

En palabras de Schwab, (2016) la Cuarta Revolución Industrial, a partir de la biotecnología hasta la inteligencia artificial, le da un nuevo sentido al significado del ser humano. Lo anterior señala que se presentarán cambios en diferentes 
aspectos del individuo como la longevidad; existe un estimativo de aumentar los años de vida debido a la mejora en la nutrición, los cuidados médicos, la educación (Gratton y Scott, 2017); la salud, referido a programas de prevención (Robledo, 2017), la forma de relacionarse entre los individuos, haciendo uso de las redes sociales (Stalman, 2018); en esta misma línea Echeverría y Martínez (2018) sostienen que las personas se deben adaptar a estos cambios y a otros similares que se darán como resultado de esta revolución.

El panorama aquí descrito puede presentar una realidad tecnológica algo distante de las aulas de tecnología en Colombia; que se limita a abordar la mayor parte de las tecnologías 4.0 solo de manera informativa; para conocer el estado de la cuestión, a continuación, se hará un recorrido histórico y descriptivo de la inclusión de la tecnología en los planes de estudios de la educación media en Colombia.

\section{Panorama histórico de la Tecnología en la educación colombiana}

Si bien el primer programa de Ingeniería de Sistemas llegó a Colombia en el año de 1967 (Universidad de los Andes, 2016) y a partir de éste se crearon diferentes programas similares en el país; en el caso de la educación media en Colombia, hubo un retraso de más de 20 años, para la inclusión del área de Tecnología e informática en los planes de estudios obligatorios.

El decreto 45 del Ministerio Nacional de Educación (1962), en su artículo noveno, establece que los estudiantes de secundaria deben cursar una asignatura denominada Artes Industriales y Educación para el Hogar, en la que los jóvenes debían formar destrezas y habilidades en actividades como mecanografía, fotografía, encuadernación, aeromodelismo, mecánica, carpintería, radio, actividades agrícolas, juguetería, modelado, culinaria o primeros auxilios; la institución educativa debía elegir una o varias de estas modalidades de acuerdo a la orientación que ofrecieran.

Para ese entonces, estas modalidades pudieron ser pertinentes debido al desarrollo tecnológico e industrial que se presentaba en el país en la época; sin embargo, el plan de estudios obligatorio establecido por el decreto 45 (Ministerio de Educación Nacional, 1962), permaneció en rigor hasta el año 1994, cuando 
aparece la ley general de educación; salvo para los Institutos Nacionales de Educación Media Diversificada (INEM) quienes mediante el decreto 363 (Ministerio de Educación Nacional, 1970), adoptan la formación vocacional en forma diferenciada por género, en donde los hombres podían optar por economía agrícola y conservación de recursos naturales, horticultura y animales domésticos, dibujo, electricidad, metalmecánica, información ocupacional, carpintería o mecanografía; mientras que las mujeres podían optar por economía agrícola y conservación de recursos naturales, horticultura, puericultura, dibujo, metalmecánica, confección información ocupacional o mecanografía.

Posteriormente, el decreto 1419 (Ministerio de Educación Nacional, 1978), crea una modalidad de bachillerato vocacional en tecnología que se puede aplicar a las modalidades pedagógica, industrial, agropecuaria, comercial, salud y nutrición, educación física y recreación o promoción de la comunidad, que las instituciones educativas podían ofrecer con base en las necesidades sociales, económicas y culturales de la región; todas estas opciones aún se encontraban al margen de la informática y de los avances en este campo que ya estaban en auge en muchas zonas del mundo.

Para el año 1990 se conforma RIBIE-Col (Red Iberoamericana de Informática Educativa - Nodo Colombia) en el marco del cumplimiento del acuerdo CYTED (programa iberoamericano de ciencia y tecnología para el desarrollo) cuyo objetivo es contribuir al desarrollo armónico de la región, mediante la cooperación para lograr resultados científicos y tecnológicos que sean transferibles al sistema económico y social (CYTED, 2020); mediante esta institución se busca promover y financiar la investigación en ciencia y tecnología para lograr el desarrollo del país.

El Departamento Nacional de Planeación lanza el Plan de Apertura Educativa 1991-1994 (1991) en el que plantea como una de las acciones prioritarias el mejoramiento de la calidad educativa, mediante la captación y sistematización de nuevas tecnologías para adaptarlas a las condiciones específicas de los procesos educativos locales; para ello, se debían incorporar los desarrollos de la informática a la educación, así como diseñar, producir y difundir, los medios audiovisuales como apoyo pedagógico. 
Con el objetivo de lograr el propósito antes descrito, se creó el Sistema Nacional de Informática Educativa (SISNIED), que buscaba propiciar en las instituciones participantes, un proceso de innovación educativa, apoyado en el uso de la tecnología, asumiendo la informática como recurso de educación; esta iniciativa tuvo un tiempo de ejecución de cuatro años, en 59 aulas de informática para 6 Centros Regionales de Informática Educativa (CRIES) y 53 Centros de Innovación en Informática Educativa (CIIES), tiempo durante el cual se hizo capacitación docente en tecnología y clarificación de modelos pedagógicos para articular la informática al currículo; durante el proceso se logró la dotación de recursos informáticos para las instituciones seleccionadas, la capacitación del personal docente y directivo, así como el acceso de los estudiantes a los recursos; en contraste, la falta de continuidad del proyecto, así como la rápida obsolescencia de los equipos suministrados fueron las principales dificultades que debió sortear el SISNIED (Universidad de los Andes, 2018).

La ejecución del proyecto del SISNIED, dejó en claro el potencial que tenían los recursos informáticos para el aprendizaje y la necesidad de invertir en estos elementos que, para ese entonces, solo estaban al alcance de universidades 0 ciertos colegios del sector privado.

En 1994, el Congreso de Colombia aprueba la ley 115, denominada Ley General de Educación (1994), que en el artículo 23 establece cómo área obligatoria y fundamental de enseñanza en el ciclo de básica y media el área de Tecnología e Informática, incluyéndola en el currículo, de todas las instituciones educativas tanto públicas como privadas y adicionando de éste modo, los equipos informáticos como elemento indispensable para el proceso educativo.

Pese al obligatorio cumplimiento de la norma antes mencionada, el proceso de dotación de equipos informáticos para las instituciones educativas fue lento y discontinuo; apenas en el año 2000 se crea el programa Computadores Para Educar (CPE), que recibía equipos de cómputo donados por particulares, los reparaba y los entregaba a las escuelas (Computadores Para Educar, 2016); desafortunadamente, las donaciones recibidas, eran principalmente de computadores con uno o más lustros de uso y su puesta a punto además de ser 
costosa, era ineficaz por la obsolescencia de las máquinas.

Debido a la situación antes descrita, a partir del año 2013, el programa CPE sólo reacondicionó equipos con procesador de doble núcleo; las demás donaciones se aprovechan mediante el reciclaje de los desechos electrónicos para obtener recursos para la compra de nuevos equipos (SITEAL, 2014).

En la actualidad el programa CPE entrega en su mayoría equipos nuevos ya sean computadores o tabletas y cuenta con un presupuesto público e ingresos por el aprovechamiento de residuos tecnológicos; por tanto, se ocupa de retomar los equipos que necesitan ser reemplazados (Computadores Para Educar, 2019); pese a lo anterior, aún no ha sido posible alcanzar la meta de un equipo por cada dos estudiantes.

Definida la obligatoriedad de la enseñanza de Tecnología e Informática en la educación media y poniendo al alcance de los estudiantes recursos para el aprendizaje de la misma, es necesario reflexionar acerca de las metas educativas que se persiguen en el proceso; a continuación, se hará un análisis de lo que se enseña actualmente en esta área.

\section{Los contenidos del programa de tecnología e informática}

En la actualidad el área de tecnología e informática, como la mayoría de asignaturas fundamentales, cuentan con un listado de estándares básicos emitidos por el propio Ministerio de Educación, en donde se especifican las competencias que los estudiantes deben alcanzar según el ciclo de enseñanza.

Los estándares del área de Tecnología e Informática, tienen su origen en el Programa de Educación para la Tecnología, PET21 (Ministerio de Educación Nacional, 1996), que era una propuesta que pretendía superar los enfoques educativos en tecnología que la relacionaban con actividades manuales o la preparación para artes u oficios y dirigirla a la identificación y solución de problemas del entorno.

Desde el PET21, se consideraba que la enseñanza de Tecnología e Informática, tenía la finalidad de desarrollar habilidades para búsqueda, manejo,

procesamiento y utilización eficiente de la información y que esto no necesariamente requería conocimientos relativos al uso de equipos de cómputo ni 
acceso a la internet; según el PET21 "resulta indispensable enfrentar con serenidad la fiebre de compra de computadores, equipos y redes informáticas; acceder a ellos no significa acceder a la tecnología" (p.13); desde luego, las habilidades que se planteaban en cuanto al manejo de información son fundamentales, sin embargo, intentar introducir el desarrollo tecnológico al país por medio de la educación, sin querer invertir en recursos tecnológicos resulta ilógico por decirlo de alguna manera; esta concepción desde el propio Ministerio de Educación, retrasó desde sus inicios la alfabetización tecnológica necesaria para el desarrollo del país e hizo que la infraestructura tecnológica de las instituciones educativas públicas fuera por mucho tiempo mínima e incluso inexistente.

La publicación de los primeros estándares para el área de Tecnología e Informática ocurrió en el año 2006, mediante un documento llamado 'Ser competente en tecnología. Una necesidad para el desarrollo' (Ministerio de Educación Nacional, 2006); este escrito incluía los fundamentos conceptuales aportados por el PET21, además, contenía la alfabetización tecnológica como un componente fundamental del derecho a la educación; este documento estableció un listado de estándares organizado en cuatro componentes, que podían verificarse mediante una lista de indicadores por componente; los estándares venían organizados por niveles: de primero a tercero, de cuarto a quinto, de sexto a séptimo, de octavo a noveno y de décimo a once.

Para todos los niveles, se establecieron cuatro componentes: naturaleza y conocimiento de la tecnología, aplicación y uso de la tecnología, solución de problemas con tecnología y por último, tecnología y sociedad; los jóvenes que finalizaban grado once, según el estándar, debían ser capaces de interpretar la tecnología como una elaboración cultural, seleccionar y utilizar eficientemente soluciones tecnológicas, identificar, formular y resolver problemas usando su conocimiento tecnológico y analizar las implicaciones éticas, sociales y ambientales de la tecnología (Ministerio de Educación Nacional, 2006).

Posteriormente, en el año 2008, se publicó la guía 30 'Ser competente en Tecnología' (Ministerio de Educación Nacional, 2008); este documento salvo 
algunos detalles de simplificación en la redacción de los estándares, disminución de la cantidad de indicadores y ediciones en el formato, gráficas y colores del documento, es similar a su antecesor; luego no se presentan modificaciones sustanciales en los estándares.

Desde entonces no se han presentado modificaciones en las competencias para el área de Tecnología e informática, así que los contenidos que se desarrollan dentro de la misma, están sujetos al criterio de la institución educativa o al docente que la orienta, ya que los estándares son muy amplios; en razón de ello, suelen adaptarse a la infraestructura tecnológica disponible y a los conocimientos del docente, que no en todos los casos es el especialista en el área; en ocasiones la enseñanza de tecnología, es asumida por docentes cuya especialidad corresponde a otras áreas, pero que tienen disponibilidad en su asignación académica.

Otro factor influyente en los contenidos que se desarrollan en el área de tecnología e informática, es la frecuencia con que suele capacitarse el docente titular del área; los desarrollos tecnológicos por lo general suelen se presentarse a diario, lo que supone una rápida obsolescencia de los conocimientos técnicos en esta materia; por tanto, mantenerse actualizado implica un estudio y capacitación continua; desafortunadamente, para el docente al servicio del estado en Colombia, la capacitación debe ser asumida por su propia cuenta y riesgo, lo que en algunos casos representa invertir recursos que no tendrán ningún retorno desde el punto de vista salarial; en contraste, en la actualidad es posible capacitarse utilizando recursos online de muy bajo costo; desde luego, esto se encuentra supeditado a la conectividad y recursos personales de hardware de que se dispongan.

Por consiguiente, el área de Tecnología e Informática, se dedica a la enseñanza de herramientas ofimáticas que el estudiante emplea para la presentación de trabajos de otras asignaturas, el manejo de las herramientas básicas de internet, la búsqueda de información en la web para ampliar sus conocimientos en determinados temas y el uso de recursos como las redes sociales de una manera responsable; si bien estos conocimientos les permiten desarrollar actividades personales, académicas o laborales que son cotidianas, los 
convierten en consumidores de recursos tecnológicos cuya habilidad es distinguir cuál es la herramienta tecnológica más apropiada para cierta tarea y utilizarla acertadamente y responsablemente; dejando de lado la posibilidad de mejorar la herramienta o producir una nueva.

Estudios en torno al uso que los jóvenes hacen en la actualidad de los recursos tecnológicos (Petrillo, De Felice, Cioffi y Zomparelli, 2018; Crovi Druetta, 2017; (World Economic Forum, 2018; Eberhard et al., 2017; Lubis, Absah y Lumbanraja, 2019; Frey y Osborne, 2013; Dombrowski y Wagner, 2014; Sook Young, 2018; Alexander et al., 2019; Zambrano Garza, Habib Mireles y Alfaro Cázares, 2019; Suganya, 2017; Flores Alanis, Chavez Gonzalez y Rodriguez Hernandez, 2018; Galindo Arranz, Ruiz Blanco y Ruiz San Miguel, 2017), permiten concluir que se requiere mayor formación en cuanto a producción tecnológica se refiere, para dejar de ser usuarios y migrar a convertirse en creadores.

Frente a éste panorama, es claro que en la actualidad el área de Tecnología e Informática en el ciclo de educación media en Colombia enfrenta un desafío relacionado con la Cuarta Revolución Industrial y lo que ésta exige a los jóvenes, en contraste con lo que se enseña en la actualidad; a continuación, se analizará el reto que implica introducir tecnologías 4.0, partiendo de la situación presente.

\section{Tendencias de la enseñanza en Tecnología e informática}

A partir de lo anterior, se pone en evidencia una grave diferencia entre los fines que el sistema educativo colombiano persigue en la actualidad desde el área de Tecnología e Informática y las exigencias de la cuarta revolución industrial.

Si bien últimamente se han planteado políticas públicas desde el Ministerio de las Tecnologías de la Información y de las Comunicaciones (MinTIC) con las que se pretende preparar al país para la $4 \mathrm{RI}$, tales como la designación de la ciudad de Medellín como Centro para la Cuarta Revolución Industrial (MinTic, 2019) o proyectos como 'Crea Digital' que es una convocatoria para coproducir contenidos digitales con énfasis culturales y educativos, el proyecto de programación para niños que busca potencializar las capacidades de los niños de colegios públicos para crear programas de computadora y que actualmente 
beneficia cerca de quince mil estudiantes, el programa 'Mejor Conectividad' para el sector rural busca incrementar el $30 \%$ de las poblaciones rurales conectadas y 'Tecnólogos 4.0' que en convenio con diferentes plataformas educativas intenta capacitar tecnólogos en áreas de cuarta revolución industrial (MinTIC, 2019), el Ministerio de Educación y en general el sistema educativo público, no ha tomado acciones globales o concretas para asumir el reto que implica ajustar la educación a la 4RI.

Según Guerrero (2004), los sistemas educativos actuales se enfocan en aspectos técnicos como la cobertura y la dotación tecnológica y dejan de lado elementos fundamentales como el currículo y lo que significa la educación en un mundo globalizado; esto puede generar la inclusión de tecnologías 4.0 en las aulas sin que el uso de las mismas vaya más allá de su implementación como elemento de mediación pedagógica.

Por consiguiente, en el área de Tecnología e Informática, las habilidades que alcanzan los estudiantes, los convierten en usuarios de tecnologías 4.0; en esta misma mirada, siguiendo a Arias Murillo (2007) sostiene que "pueblo o sociedad que no produce ni invierte en la producción de tecnología está obligado a importarla"(p.6); en la situación actual, el país siempre será consumidor de desarrollos tecnológicos importados, que requerirán cierto grado de capacitación por parte de sus operarios, así que la función del sistema educativo estaría orientada hacia la instrucción para su uso.

Desde luego, está en manos del propio sistema educativo la tarea de repensar los fines de la educación tecnológica en Colombia; asimilar que ya no basta con la alfabetización en este campo; sino que por el contrario, el desarrollo de las comunidades y desde luego del país, depende de la producción tecnológica que se alcance, mas aún en situaciones como la actual; por tanto, es urgente tomar acciones que permitan dar respuesta a las demandas del contexto, en donde las habilidades tecnológicas permitan a los individuos transformar su entorno, adaptando a sus necesidades las soluciones existentes o creando nuevas soluciones hechas a la medida.

Replantear las metas educativas del área de Tecnología e Informática, no 
tendría sentido, sin evaluar en detalle la congruencia de las mismas con el proceso que se desarrolla al interior de las aulas y los medios que se requieren para alcanzarlas; es necesario que el Estado asuma el mejoramiento de la infraestructura tecnológica de las instituciones educativas, no como un gasto, sino como una inversión cuyo retorno se verá reflejado en el incremento del capital humano y el desarrollo tecnológico del país.

También es necesario que el personal docente (no solamente los del área de tecnología e Informática) asuma el reto de capacitarse constantemente, en especial en lo relativo a tecnologías 4.0 y habilidades de cuarta revolución industrial; aunque poner al alcance de los profesores continuamente programas de capacitación, sea una función del gobierno (Presidencia de la República de Colombia, 2002), la capacidad de autoaprendizaje y el deseo de mejorar cada día en su labor, son características propias del maestro que le permiten desarrollar su labor con calidad y su propia formación debe ser una prioridad.

El desafío de la inclusión de las tecnologías 4.0 en las aulas de Tecnología e Informática de la educación media colombiana, será un reto que se enfrentará en diferentes frentes: desde lo político a través de la definición de normas y procedimientos que permitan la transición; en lo económico mediante la destinación de recursos necesarios para su implementación, desde lo académico impulsando la transformación curricular que requiere; en lo social mediante la eliminación de las brechas tecnológicas y de conectividad, en lo cultural mediante la generación de nuevas formas de ver e interpretar la realidad y en la práctica mediante la apropiación que cada miembro de la comunidad educativa haga del proceso; para finalizar, podría decirse que el desafío debe asumirse de manera inmediata, con el fin de evitar el atraso del sistema escolar frente a la realidad que se exige en este momento.

Por consiguiente, desde la mirada de Pérez y Hernández (2018) las TIC se ligan a los procesos de enseñanza-aprendizaje en todos los niveles de formación, es así que en este nuevo siglo XXI, la tecnología se implementa de manera transversal, en cada una de las áreas del conocimiento, esto significa que hay una responsabilidad por parte de los docentes, en consolidar proyectos de integración 
como oportunidad para fortalecer aspectos cognoscitivos, sociales y afectivos a través del trabajo en equipo como estrategia de aprendizaje, con lo cual se busca lograr mejores habilidades sociales, desarrollar la argumentación y el respeto por las ideas, además se resalta la importancia del trabajo en equipo como la manera de descubrir el camino para el logro de las metas trazadas de los participantes, lo importante en estos espacios de encuentro y de reflexión es que prime la confianza y el apoyo recíproco entre todos (Ahumada Méndez...et al, 2019).

Siguiendo a Rueda y Quintana (2013), las instituciones educativas deben estar a la vanguardia con los cambios culturales y tecnológicos del momento actual, es así, que la revolución tecnológica exige el dominio de habilidades de procesamiento y uso de la información. Lo anterior, significa que el sistema educativo necesita la integración del uso de las Tecnologías 4.0 en los procesos de enseñanza y aprendizaje, por lo tanto, los docentes deben conocer y apropiar esta competencia y estar actualizados en los avances de la tecnología. Desde luego, en la orientación requerida en la Revolución 4.0, se deben desarrollar competencias para identificar, seleccionar y reorientar alternativas individuales, académicas y profesionales, a nivel personal, contrastadas con las ofertas de los entornos formativos y sociolaborales.

\section{Referencias}

Ahumada Méndez, L.S.(comp.). (2019). Estrategias de enseñanza aprendizaje: una mirada desde la investigación. Bogotá: Ediciones Universidad Cooperativa de Colombia.

Alexander, B., Ashford-Rowe, K., Barajas-Murphy, N., Dobbin, G., Knott, J., McCormack, M., y otros. (2019). EDUCAUSE Horizon Report / 2019 Higher Education Edition. Louisville -USA: EDUCAUSE.

Alonso - Calpeño, M., Santander - Castillo, J., Ramirez - Chocolatl, Y., \& Alanis Teutle, R. (2019). Cómputo en la niebla aplicado a la manufactura inteligente bajo el contexto de la industria 4.0: desafíos y oportunidades. México: ECORFAN.

Arias Murillo, F. (2007). Educación en la globalización: un cambio en la perspectiva. Revista Latinoamericana de Ciencias Sociales, Niñez y Juventud , 5 (1), 1-18. 
Bauer, W., Schlund, S., Marrenbach, D., \& Ganschar, O. (2014). Industrie 4.0 Volkswirtschaftliches Potenzial für Deutschland. BITKOM , 5-43.

Berchon, M., \& Luyt, B. (2016). La impresión 3D. Guía definitiva para makers, diseñadores, estudiantes, profesionales, artistas y manitas en general . Barcelona: Editorial Gustavo Gili S.L.

C Glenn, J., Florescu, E., \& Millenium Project Team. (2016). 2015-16 State of the Future. Journal of Socialomics , 5 (3), 1-7.

Castañares, W. (2011). Realidad virtual, mímesis y simulación. CIC Cuadernos de Información y Comunicación, 16, 59-81.

Castillo, J. (2018). Big Data. IFCT128PO. Málaga: IC Editorial.

Celaya, A. (2014). Cloud: Herramientas para trabajar en la nube. Málaga: ICB, S.L. Computadores Para Educar. (2016). Historia. Recuperado el 7 de Agosto de 2020, de computadoresparaeducar.gov.co:

https://www.computadoresparaeducar.gov.co/es/historia

Computadores Para Educar. (2019). Informe de gestión 2018. Bogotá: Computadores Para Educar.

Congreso de Colombia. (1994). Ley 115 de 1994. Bogotá: Congreso de Colombia. Crovi Druetta, D. (2017). Estudiantes ante la apropiación de espacios digitales. En P. Avila Muñoz, \& C. Rama, Internet y educación: amores y desamores (págs. 131-153). México: INFOTEC Centro de Investigación e Innovación en Tecnologías. CYTED. (2020). Programa Iberoamericano de Ciencia y Tecnología para el Desarrollo. Recuperado el 7 de Agosto de 2020, de cyted.org: http://www.cyted.org/es/Objetivos

Departamento Nacional de Planeación. (1991). Plan Nacional de Apertura Educativa (1991-1994). Bogotá: Departamento Nacional de Planeación.

Dombrowski, U., \& Wagner, T. (2014). Mental strain as field of action in the 4th industrial revolution. ScienceDirect, 100-105.

Eberhard, B., Podio, M., Perez Alonso, A., Radovica, E., Avotina, L., Peiseniece, L., y otros. (2017). Smart Work: The Transformation of the labour market due to fourth industrial revolution. International Journal Bussines and Economic Sciences Aplied Research , 10 (3), 47-66. 
Echeverría Samanes, B. Martínez Clares, P. (2018) Revolución 4.0, competencias, educación y orientación. Revista digital de investigación en Docencia Universitaria. Equipo Vortice. (2010). Técnicas Avanzadas de Diseño Web. Málaga: Ediciones Vórtice.

Flores Alanis, A., Chavez Gonzalez, G., \& Rodriguez Hernandez, J. (2018). Educacion 4.0: Transformación del aula tradicional en las escuelas de educación básica en México. Conference Proceedings EDUNOVATIC 2018: 3rd Virtual International Conference (págs. 144-147). Eindhoven - Holanda: EDUNOVATIC.

Frey, C., \& Osborne, M. (2013). The future of employment: how susceptible are jobs to Computerisation? Oxford -UK: Oxford University.

Galindo Arranz, F., Ruiz Blanco, S., \& Ruiz San Miguel, F. (2017). Competencias digitales ante la irrupción de la Cuarta Revolución Industrial. Estudos em Comunicação, 1 (25), 1-11.

Gonzalez Garcia, M. (2018). Definición de estrategias de adopción de la cuarta revolución industrial por parte de las empresas en Bogotá, aplicables a pymes en Colombia. Bogotá: UNIVERSIDAD CATÓLICA DE COLOMBIA.

Guerrero, G. (2004). La educación en el contexto de la globalización. Rhela , 6, 343-354.

Gratton, L., \& Scott, A. (2017). La vida de 100 años: Vivir y trabajar en la era de la longevidad. Málaga: Lettera Publicaciones, S.L.

Hecklau, F., Flachs, S., \& Khol, H. (2016). Holistic Approach for Human Resource Management in Industry 4.0. Procedia CIRP , 1-6.

Hiemenz, J., \& Stratasys, I. (2011). La impresión 3D con FDM. ¿Como Funciona? Rheinmüster - Alemania: Stratasys Europe.

Householder, B. (2018). A mix of hope an d ambiguity. The fourth industrial revolution si here, Are you ready? Deloitte Insights , 2-4.

La Nación. (26 de Octubre de 2017). Estas son las 50 aplicaciones más populares en Android. Recuperado el 7 de Abril de 2020, de lanacion.com.ar: https://www.lanacion.com.ar/tecnologia/estas-son-las-50-aplicaciones-maspopulares-en-android-nid2076553 
Liao, Y., Rocha Loures, E., Deschamps, F., Brezinski, G., \& Venâncio, A. (2018). The impact of the fourth industrial revolution: a cross-country/region comparison. Production , 28, 1-18.

Lubis, A., Absah, Y., \& Lumbanraja, P. (2019). Human resource competencies 4.0 for generation z. European Journal of Human Resource Management Studies , 3 (1), 95-105.

Manpower Group. (2018). Solving the Talent Shortage Build, Buy, Borrow and Bridge. Milwaukee- USA: Manpower Group.

Mantaras, R., \& Meseguer, P. (2017). Inteligencia Artificial. Madrid: CSIC.

Mantilla Avendaño, L., \& Gonzalez-Perez, M. (2015). Industria 5.0: ¿Vuelve el hombre al centro de los procesos de producción? Medellín: Universidad EAFIT.

Manyika, J., Chui , M., Miremadi, M., Bughin, J., George, K., Willmott, P., y otros. (2017). A future that works: automation, employment, and productivity. New YorkEUA: McKinsey \& Comapany.

Miller, D. (2016). Natural Language: The User Interface for the Fourth Industrial Revolution. Opusresearch Report , 3-7.

Ministerio de Educación Nacional. (1962). Decreto 45v de 1962. Bogotá: Ministerio de Educación Nacional.

Ministerio de Educación Nacional. (1970). Decreto 363 de 1970. Bogotá: Ministerio de Educación Nacional.

Ministerio de Educación Nacional. (1978). Decreto 1419 de 1978. Bogotá: Ministerio de Educación Nacional.

Ministerio de Educación Nacional. (1996). Educación en Tecnología: Propuesta para la educación básica. Bogotá: Ministerio de Educación Nacional.

Ministerio de Educación Nacional. (2006). Estándares básicos de competencias en Tecnología e Informática. Bogotá: Ministerio de Educación Nacional.

Ministerio de Educación Nacional. (2008). Guía 30. Ser competente en tecnología. Bogotá: Ministerio de Educación Nacional.

Ministerio de Tecnologías de la Información y las Comunicaciones. (20 de Abril de 2019). Colombia en la Cuarta Revolución Industrial. Recuperado el 21 de Agosto de 2020, de mintic.gov.co: https://www.mintic.gov.co/portal/inicio/Sala-de- 
Prensa/Columnas-Ministra-TIC/100434:Colombia-en-la-Cuarta-RevolucionIndustrial

Ministerio de Tecnologías de la Información y las Comunicaciones. (10 de Mayo de 2019). Conozca cómo es el Centro para la Cuarta Revolución Industrial en el quinto programa de 'Más TIC, Mejor País'. Recuperado el 21 de Agosto de 2020, de minitc.gov.co: https://www.mintic.gov.co/portal/inicio/Sala-dePrensa/Noticias/100580:Conozca-como-es-el-Centro-para-la-Cuarta-RevolucionIndustrial-en-el-quinto-programa-de-Mas-TIC-Mejor-Pais Minsky, M. (2010). La máquina de las emociones: Sentido común, inteligencia artificial y el futuro de la mente humana. Barcelona: Random House Mondadori. Morrar, R., Arman, H., \& Mousa, S. (2017). The Fourth Industrial Revolution (Industry 4.0): A Social Innovation 12 Perspective. Technologu Innovation Management Review , 7 (11), 12-20.

Navarro, F., Martinez, A., \& Martinez, J. (2018). Realidad virtual y realidad aumentada. Desarrollo de aplicaciones. Madrid: Ra-Ma Ediciones.

Parés, N., \& Parés, R. (2010). Realidad Virtual. (E. UOC, Ed.) Barcelona.

Petrillo, A., De Felice, F., Cioffi, R., \& Zomparelli, F. (2018). Challenges, and Opportunities Fourth Industrial Revolution: Current Practices, Challenges, and Opportunities. INTECH, 1-20.

Pérez, E., \& Hernández, E. (2018). La orientación educativa en las prácticas inclusivas de la educación superior cubana. Psicologia Escolar e Educacional , 7785.

Prendes Espinosa, C. (2015). Realidad Aumentada y Educación: Análisis de Experiencias Prácticas . Píxel-Bit. Revista de Medios y Educación. (46), 187-203.

Presidencia de la República de Colombia. (2002). Decreto 1278 de 2002. Bogotá: Presidencia de la República de Colombia.

ProArgentina. (2005). Biotecnología. Buenos Aires: Ministerio de Economía y Producción. República Argentina.

Reyes, F. (2011). Robótica. Control de robots manipuladores. Méxido: Alfaomega Grupo Editor, S.A. de C.V. 
Robledo, R. (2017). Revolución Industrial en el sector Salud. ¿Regreso al futuro? Recuperado de https://goo.gl//bsk7kD

Rodríguez, G. (1998). Ciencia, Tecnología y Sociedad: una mirada desde la Educación en Tecnología. Revista Iberoamericana de Educación , 107-143.

Rojko, A. (2017). Industry 4.0 Concept: Background and Overview. International Journal of Interactive Mobile Technologies , 11 (5), 77-90.

Romero, D., Stahre, J., Wuest, T., Noran, O., Bernus, P., Fast-Berglund, A., y otros. (2016). Towards an operator 4.0 typology: A human-centric perspective on the fourth industrial revolution technologies. Proceedings , 1-11.

Rose, G. (2016). The Fourth Industrial Revolution: A Davos re- ader. New York: Council on Foreign Relations.

Salgado, R. (2019). Vehículos Autónomos ¿Un vehículo que se conduce solo? Recuperado el 9 de Abril de 2020, de sabermas.umich.mx: https://www.sabermas.umich.mx/archivo/tecnologia/196-numero-2462/381-

vehiculos-autonomos-iun-vehiculo-que-se-conduce-solo.html

Rueda Rocio, Quintana Antonio (2013). Ellos vienen con el chip. Serie de Investigación IDEP.Editorioal Jotamar. Bogotá.

Santamaría-Puerto, G., \& Hernández-Rincón, E. (2015). Aplicaciones Médicas Móviles: definiciones, beneficios y riesgos. Revista Científica Salud Uninorte , 3 (3), 599-607.

Stalman, A. (2018). Humanoffon: ¿Está internet cambiándonos como seres humanos?. Bilbao: Deusto Ediciones.

Schwab, K. (2016). The fourth industrial revolution. New York: World Economic Forum.

SITEAL. (2014). SITEAL/TIC. Recuperado el 7 de Agosto de 2020, de tic.siteal.iipe.unesco.org/:

http://www.tic.siteal.iipe.unesco.org/politicas/882/computadores-para-educar

Sook Young, C. (2018). A Study on the Digital Competency for the Fourth Industrial Revolution. Revista de la Sociedad de Educación Informática de Corea , 21 (5), 25-35.

Stoppa, M., \& Chiolerio, A. (2014). Wearable Electronics and Smart Textiles: A Critical Review. Sensors, 11957-11992. 
Suganya, G. (2017). A Study on Challenges before Higher Education in the Emerging Fourth Industrial Revolution. IJETSR - International Journal of Engineering Technology Science and Research , 4 (10), 1-3.

Taniguchi, N. (1974). On the Basic Concept of Nanotechnology. Tokio: Actas de la Internacional Conference on Production.

Tapscott, D., \& Tapscott, A. (2017). La Revolución Blockchain. Barcelona: Grupo Planeta.

UNESCO. (2005). Competency Framework. París: UNESCO.

Universidad de los Andes. (2016). Pregrado en Ingeniería de Sistemas y Computación ISIS. Recuperado el 7 de Agosto de 2020, de uniandes.educ.o: https://sistemas.uniandes.edu.co/es/isis

Universidad de los Andes. (2018). Evaluación del Sistema Nacional de Informática Educativa. Revista Informática Educativa, 225-267.

World Economic Forum. (2018). The Future of Jobs Report 2018. Geneva - Suiza: World Economic Forum.

Xu, M., David, J., \& Kim, S. H. (2018). The Fourth Industrial Revolution: Opportunities and Challenges. International Journal of Financial Research , 9 (2), 90-95.

Yinghui, H., \& Guanyu, L. (2010). Descriptive Models for Internet of Things. Dalian - China: IEEE International Conference on Intelligent Control and Information Processing.

Ynzunza Cortés, Carmen Berenice; Izar Landeta, Juan Manuel; Bocarando Chacón, Jacqueline Guadalupe; Aguilar Pereyra, Felipe; Larios Osorio, Martín El Entorno de la Industria 4.0: Implicaciones y Perspectivas Futuras Conciencia Tecnológica, núm. 54, 2017 Instituto Tecnológico de Aguascalientes, México Disponible en: http://www.redalyc.org/articulo.oa?id=94454631006

Zambrano Garza, M., Habib Mireles, L., \& Alfaro Cázares, N. G. (2019). Educación superior en el contexto de la industria 4.0. Acapulco: XV Congreso Nacional de Investigación educativa. 